\title{
Water pollution monitoring using matched spatial filters
}

\author{
Silverio P. Almeida and James Kim-Tzong Eu
}

\begin{abstract}
Presented are some results obtained from the application of matched spatial filtering techniques to the identification of biological specimens called diatoms (i.e., water algae). A prototype semiautomatic optical processor has been developed that utilizes the Vander Lugt type complex spatial filters. We describe the optical filter averaging technique employed and discuss our semi in situ developing method for the filters. The advantage of this method with regards to the critical position requirements for time sharing optical filters is also presented. The filter holder is mounted on $X, Y$ stages and precision positioned under the control of a PDP-11-40 computer.
\end{abstract}

\section{Introduction}

In this paper we present a prototype optical processing system that is a new application of matched spatial filtering techniques applied to the identification of biological specimens called diatoms (i.e., water algae). A preliminary description of the prototype optical processor used has already been mentioned in previous papers. ${ }^{1,2}$ We shall, therefore, concentrate our discussion on

(a) the construction of matched spatial filters, including averaged filters to take into account variations in depth of focus and size,

(b) semi in situ developing and time sharing of filters,

(c) results obtained.

It is known $n^{3-5}$ that the number of diatoms found in a sample of water, counted as a function of species diversity over a period of time, can give important information on the pollution present in the water. Often times there are two or more species whose ratio of occurrence is a particularly sensitive early warning indicator for the health of the water system. At the present time, counting and classifying diatoms for diversity index curves is a tedious and time consuming process. The samples are counted under a microscope and require a highly trained person to be able to classify them objectively. The use of humans to perform this task becomes impractical when one wishes to classify continuously very large numbers of diatoms and to look at many different bodies of water. Hence, an automated or semiautomated system would be of great importance to the scientists in this field of study. The ability to be able to classify

The authors are with Virginia Polytechnic Institute and State University, Physics Department, Blacksburg, Virginia 24061.

Received 20 September 1975. objectively the diatoms itself is an important problem to solve. Since the diatoms have detailed structures that can to the unskilled person easily be confused, the subjectiveness of the counting then becomes an important bias to eliminate. We have chosen to apply matched spatial filtering techniques to solve the pattern recognition. The advantages of a parallel processor together with the ability for discriminating fine details in the diatoms led us to believe this method was worth careful study. As will be seen from the results obtained with the optical processor, matched filtering techniques are capable of identifying the diatoms. The degree of discrimination depends on the spatial frequency of the filter, its diatom orientation, the diatom's size variation, and whether the filter was averaged.

\section{Optical Processor and Matched Filtering}

For some time now, optical processing methods that make use of two-dimensional coherent or incoherent matched spatial filtering techniques have been applied to various pattern recognition problems. ${ }^{6-9}$ Perhaps the most widely referred to application is in character recognition. Success of the technique has, however, been limited, depending to a large degree on the complexity of the initial problem. There is no doubt that the achievement of a successful parallel optical processor is of great value in speeding up data acquisition and for analysis in the area of pattern recognition. The problem of pattern recognition by spatial filtering can be divided into three general areas:

(a) conversion of input into a form suitable for coherent processing;

(b) the problem of constructing a suitable complex spatial filter;

(c) the interfacing of optical apparatus and the filtered output to a computer in order to carry out realtime operations. 


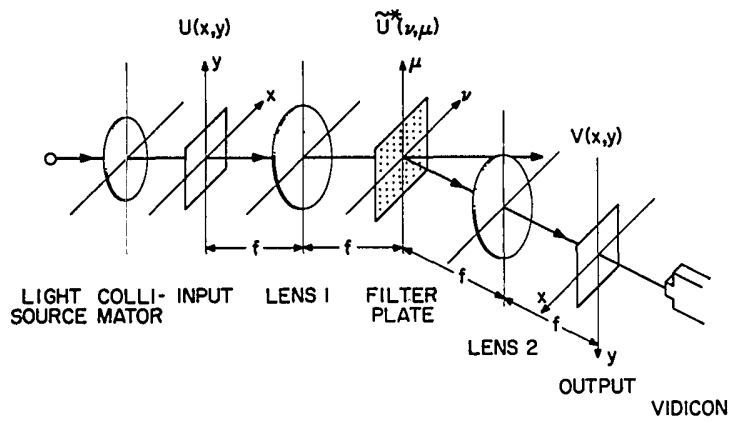

Fig. 1. Schematic diagram of the optical processor used in diatom identification.

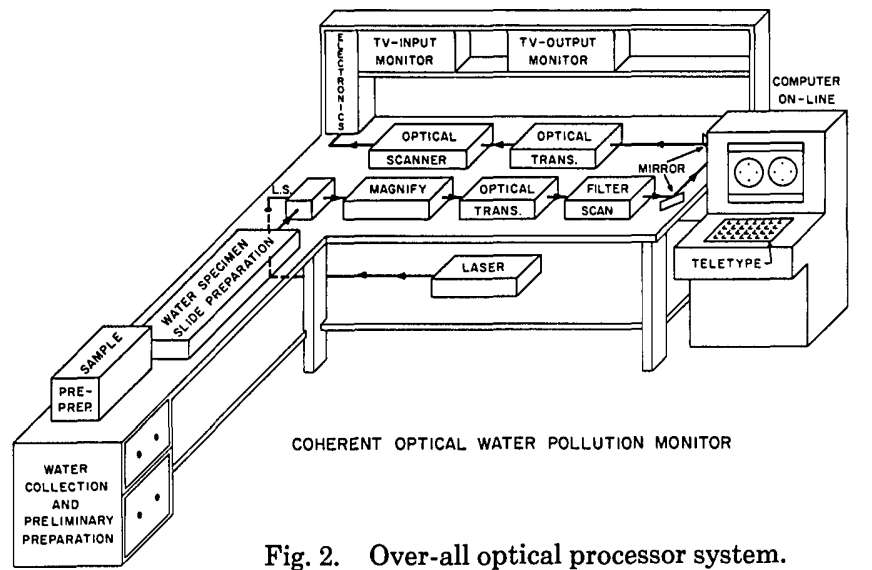

Fig. 2. Over-all optical processor system.

Success in areas (a) and (b) depend to a large extent on the particular application and in area (c) on the hardware available to the researcher as well as the state of the art of certain recording materials.

The application for the matched spatial filtering techniques that we have chosen is new in the biological area. The identification of diatoms (water algae) is well suited to this optical technique, some reasons being (a) the diatoms are rigid silicon dioxoide skeletons, (b) they are quite symmetrical about one and in some cases two axis, (c) they possess a fairly consistent uniformity among a given species, (d) on the average, size variations are about $15 \%$ of their maximum, (e) due to the diatom's detailed structure one is able to obtain a high degree of discrimination, although at the expense of a more stringent requirement on their orientation.

The use of the Vander Lugt ${ }^{6}$ type filter was employed in these studies. The problems associated with the positioning requirements of such a filter and the construction of a suitable filter for our application are now presented.

Figure 1 shows a diagram of the optical system, while Fig. 2 shows a block diagram of the over-all optical processor system. The important features of the system are: (a) the matched spatial filter plate is under computer control in the $X, Y$ directions (by typing on the console the coordinates of the $10 \times 10$ matrix filters the stages can be automatically moved to within $2.5 \mu \mathrm{m}$ for either constructing the filters or getting their correlation signals out); (b) the changing of the input (35-mm film) is also under computer control, whereby each frame is automatically ad-

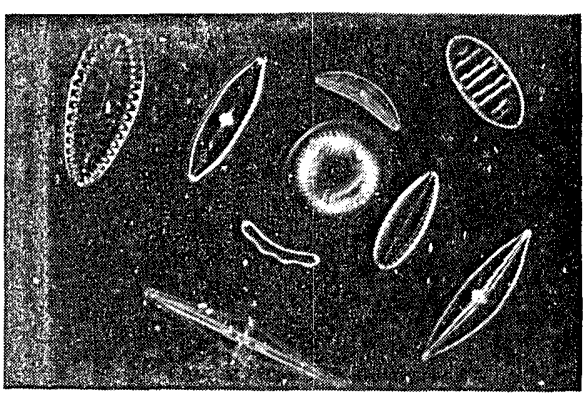

(a)

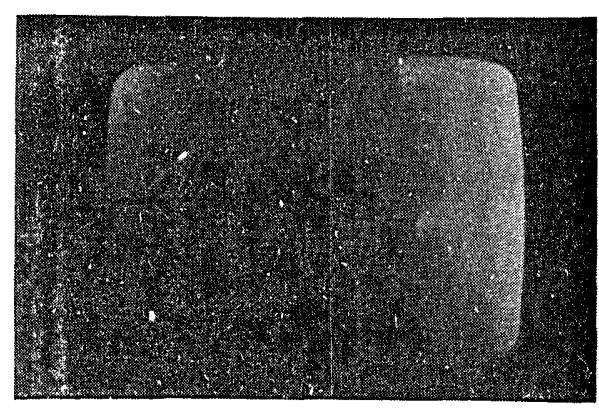

(b)

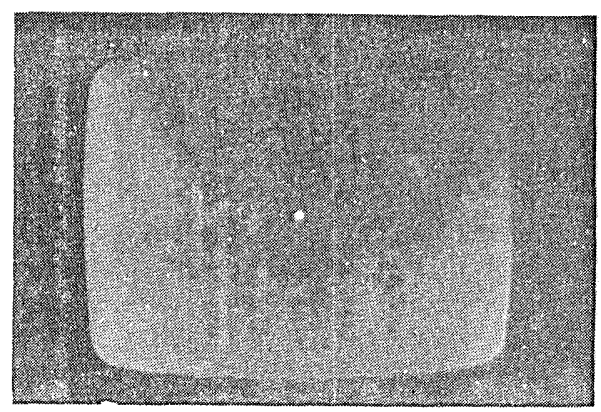

(c)

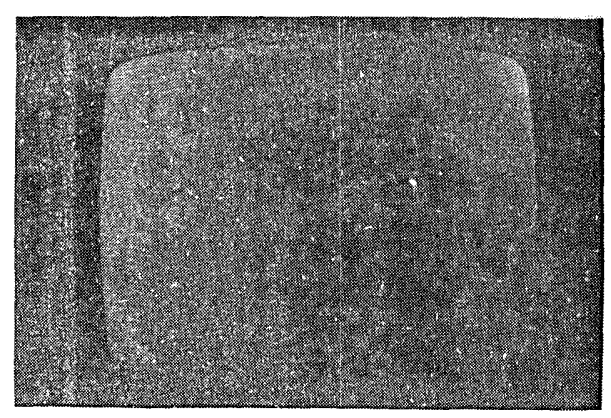

(d)

Fig. 3. (a) Input diatom patterns; (b), (c), (d) output correlation peaks of three single signals. 
vanced by a film transport; (c) the correlation signal output is received by a vidicon camera and digitized and stored in the computer for further analysis and plotting on either a storage scope or a digital plotter. The present prototype system has not yet been optimized for speed in analysis. The achievement of speed can be accomplished by substituting fäster stepping motors, film transports, and image rotators. The ultimate speed problem, as mentioned earlier, will still depend to a certain extent on the state of the art for real-time read and write imaging devices. Such real-time read and write materials as liquid crystals, thermoplastics, photochromics, and devices as the PROM ${ }^{10,11}$ can, hopefully, one day be used successfully in the input conversion and possibly in the Fourier transform planes.

\section{Filter Positioning Requirements}

As input to the optical processor we used $35-\mathrm{mm}$ positive film transparencies. The positives are made by using phase contrast microscopy techniques on prepared water sample slides. Shown in Fig. 3(a) is a photograph of some typical diatoms used as input (and also for construction of the matched spatial filters). In the processor we wish to time share the spatial filter's, that is, we have 100 filters on a 2 -in. $\times$ 2 -in. $(5-\mathrm{cm} \times 5-\mathrm{cm})$ photographic plate. This means that the positioning of the filter plate holder becomes quite critical when we want to address any one of the filters at will. To explain this positioning requirement, we use a slightly different approach from that of Vander Lugt's. ${ }^{2}$ Let the unknown input $U(x)$ consist of one of $N$ possible signals (diatoms), $U_{s 1}$, $U_{s 2} \ldots U_{s N}$. Their corresponding matched filters are $\tilde{U}_{s 1}^{*}, \tilde{U}_{s 2}^{*} \ldots \tilde{U}_{s N}^{*}$, assuming that we are matching with the particular filter $\tilde{U}_{s m}^{*}$, and that there is that particular diatom $U_{s m}$ present in the unknown input. Neglecting the cross-correlating terms, the output function $V(x)$ of the Vander Lugt matched filtering system would be

$$
V(x)=\int \tilde{U}_{s m}(\nu) \tilde{U}_{s m}^{*}(\nu) \exp (-2 \pi i \nu x) d \nu,
$$

where

$$
\tilde{U}_{s m}(\nu)=\int U_{s m}\left(x^{\prime}\right) \exp \left(-2 \pi i \nu x^{\prime}\right) d x^{\prime},
$$

and we use one-dimensional notation to simplify the calculation.

A small amount of displacement $\Delta \nu$ of the filter from its optimum position will give rise to $V(x)$ as

$$
V(x)=\int \tilde{U}_{s m}(\nu) \tilde{U}_{s m}{ }^{*}(\nu+\Delta \nu) \exp (-2 \pi i \nu x) d \nu,
$$

then, $V(x)$ can be expressed as

$$
\begin{aligned}
V(x)=\iiint U_{s m}\left(x^{\prime}\right) U_{s m} *\left(x^{\prime \prime}\right) \exp \left[2 \pi i \nu \left(-x-x^{\prime}\right.\right. \\
\left.\left.+x^{\prime \prime}\right)\right] d \nu \cdot \exp \left(2 \pi i \Delta \nu x^{\prime \prime}\right) \cdot d x^{\prime} d x^{\prime \prime} \\
=\iint U_{s m}\left(x^{\prime}\right) U_{s m} *\left(x^{\prime \prime}\right) \exp \left(2 \pi i \Delta \nu x^{\prime \prime}\right) \delta\left(-x-x^{\prime}\right. \\
\left.+x^{\prime \prime}\right) d x^{\prime} d x^{\prime \prime}, \quad \text { (3) }
\end{aligned}
$$

where we have used the relation

$$
\int \exp \left[2 \pi i v\left(-x-x^{\prime}+x^{\prime \prime}\right)\right] d v=\delta\left(-x-x^{\prime}+x^{\prime \prime}\right),
$$

and $\delta(x)$ is a Dirac delta function. Then, dropping subscription of $U_{s m}$,

$$
V(x)=\int U\left(x^{\prime}\right) U^{*}\left(x+x^{\prime}\right) \exp \left[2 \pi i \Delta \nu\left(x+x^{\prime}\right)\right] d x^{\prime} .
$$

At $x=0$, we obtain the peak intensity, which is

$$
|V(0)|^{2}=\left.\left.\left|\int_{-\frac{\Delta x}{2}}^{+\frac{\Delta x}{2}}\right| U\left(x^{\prime}\right)\right|^{2} \exp \left(2 \pi i \Delta \nu x^{\prime}\right) d x^{\prime}\right|^{2} .
$$

Where we have assumed that $\left|U\left(x^{\prime}\right)\right|^{2} \neq 0$ only in $\left|x^{\prime}\right|$ $\leq(\Delta x / 2), \Delta x$ is the object width, and we have ignored the normalization factor. The distortion phase $\phi=$ $2 \pi \Delta \nu \cdot x^{\prime}$ is then given by $|\phi| \leq \pi \Delta x \cdot \Delta \nu$. We assume (based on the precision of our apparatus) that the tolerable phase error can be equal to or less than $\pi / 4$. Then we have

$$
|\phi| \leq \pi \Delta x \Delta \nu \leq(\pi / 4),
$$

or $\Delta \nu \leq(1 / 4 \Delta x)$.

Since $\nu=\xi / \lambda f$, where $\xi$ is the spatial coordinate in the frequency domain,

$$
\begin{gathered}
\Delta \xi=\lambda f \Delta \nu \text { or } \\
\Delta \xi \leq[\lambda f /(4 \Delta x)] .
\end{gathered}
$$

We have related $\Delta \xi$, the tolerable displacement of the filter from its optimum position, to the system parameters $\lambda, f$, and $\Delta x$, where $\lambda$ is the wavelength of the laser beam, $f$ the focal length of the Fourier transform lens, and $\Delta x$ the object width at input.

The parameters we used in our optical system were $\lambda=0.63 \times 10^{-6} \mathrm{~m}(\mathrm{He}-\mathrm{Ne})$,

$f=0.60 \mathrm{~m}$,

$\Delta x=35 \mathrm{~mm}=0.035 \mathrm{~m}$.

This gives $\Delta \xi \leq 2.7 \mu \mathrm{m}$, which is within the positioning accuracy of the computer controlled stepping motors (i.e., $2.5 \mu \mathrm{m}$ ).

In order to circumvent the difficulty in time sharing the filters, we construct the filters in the form of a $10 \times 10$ matrix on a 5-cm $\times 5-\mathrm{cm}$ Kodak holographic film plate, type SO-120-02. These 100 filters are made automatically. Both the sequential stage holder positioning of the location of each individual filter and the electronic shutter for exposure are controlled by the PDP-11-40 computer. The exposed plate is then developed semi-in situ, by which we mean the plate is fixed permanently on a metal frame that undergoes development and fixation along with the plate. The frame is built in such a manner that it can be removed from the stage holder for development and then replaced within a precision of a few microns. We have achieved this, and we can snap the plate frame into the stage holder, and the change in the correlation peak intensity is negligibly small.

It is also possible to loosen the parameter requirements such that the filter positioning is not so critical. That is, one can either increase the focal length of the Fourier transform lens, or decrease the size of the input transparency, or both. For our particular needs in storage and input resolution the tolerance of about $2.5 \mu \mathrm{m}$ seems to be suitable. 
In addition to the problem of diatom identification by matched filtering, two other problems come up: (a) the question of size variation among diatoms and (b) the orientation of the diatoms. In the case of size variations, the diatoms vary, on the average, about $15 \%$ of their full size. We are able to maintain a high autocorrelation SNR via a special optical system (subject of a later paper). We are also looking into the possibility of using statistical means to account for the size variations. This latter approach would be more desirable because of the time one could save by eliminating additional filtering data. It should also be noted that the diatoms are two-dimensional projections on our input slides, and we are interested only in their front view (i.e., no side or end views). Since most of them lay flat there is little problem with taking only this view for analysis purposes. The problem of orientation, which is beyond a few degrees, can be taken into account by rotating the input. Again, the use of a statistical approach would be more desirable. In this case one could count under a microscope the diatoms of various orientations, then use this information to weigh statistically the filter used at one orientation. This approach would eliminate the need for rotating filters or diatom images. This latter method looks promising since there appears to be a random orientation to the diatoms.

\section{Averaged Filter Construction}

Due to the problems of size variation and depth of focus, some diatoms from a common species have slightly different appearance in our inputs. As a result, when an arbitrary diatom is chosen as a filtergenerator input to construct a filter for this particular diatom species, some correlation peaks at the output will become weaker. A solution to this problem is to construct an averaged filter, i.e., a filter that contains the typical possible features of a common diatom species.

In general, one can average optical signals either by coherent averaging or by incoherent averaging. By coherent averaging we mean simultaneous recording of several patterns. While by incoherent averaging, we mean sequential recording. In reconstruction (matching), both methods let the individual signals interact coherently. Coherent construction of the averaged filter could be done by essentially the same method as was described in detail by Viénot et al. ${ }^{13}$ for averaged Fourier holograms. All the patterns to be averaged are presented in the input plane together with their reference beams in a form of Dirac delta functions; the filter is realized in a single exposure followed by subsequent double diffraction to block out the unwanted intermodulation terms.

In incoherent averaging, the diatom patterns to be averaged are put in a filter-generator input plane, one at a time, then superposition of each individual exposure is carried out and results in the multiple exposures of the matched filters. We use incoherent averaging to construct our averaged filter because this method has the advantage of being much easier to realize. This is due to the fact that in the coherent case, in addition to the desired matched filter function and the usual undiffracted dc terms, there are unwanted intermodulation terms that have to be masked out to obtain the averaged signal. Hence, multiple steps are required to filter realization. Whereas, in the incoherent case, since each individual filter is constructed at a different time, they are incoherent with respect to each other. Hence, no intermodulation terms are present, and multiple diffraction steps are avoided. Besides, in the incoherent case, the same reference beam was used for all the patterns, and all the patterns in input plane located at the same coordinate (one pattern at a time). These factors further simplify the process of realizing the averaged spatial matched filter. An analysis of incoherent averaging follows.

At filter-generator input, $N$ slightly different diatom patterns to be averaged are presented one at a time, i.e., $U_{n}(x), n=1,2, \ldots N$.

At the filter plane, the complex amplitude is

$$
\tilde{U}_{n}(\nu)+\exp \left(-2 \pi i \nu_{0} x\right),
$$

where we have assumed unit amplitude for the reference beam, and $\nu_{0}$ is the carrier frequency of the reference beam.

The intensity recorded on the filter plate when pattern $n$ is presented in the input plane is given by

$$
\begin{aligned}
I_{n}= & \left|\tilde{U}_{n}(\nu)+\exp \left(-2 \pi i \nu_{0} x\right)\right|^{2} \\
=\left|\tilde{U}_{n}(\nu)\right|^{2}+1+\tilde{U}_{n}^{*}(\nu) \exp \left(-2 \pi i \nu_{0} x\right) & \quad+\tilde{U}_{n}(\nu) \exp \left(2 \pi i \nu_{0} x\right) .
\end{aligned}
$$

The total intensity of multiple exposures of all $N$ patterns is

$$
\begin{aligned}
I=\sum_{n=1}^{N}\left|\tilde{U}_{n}(\nu)\right|^{2}+C_{0}+\sum_{n=1}^{N} \tilde{U}_{n}^{*}(\nu) & \exp \left(-2 \pi i \nu_{0} x\right) \\
& +\sum_{n=1}^{N} \tilde{U}_{n} \exp \left(2 \pi i \nu_{0} x\right)
\end{aligned}
$$

where

$$
\sum_{n=1}^{N} U_{n}^{*}(\nu) \exp \left(-2 \pi i \nu_{0} x\right)
$$

is the desired matched filter function, and $C_{0}$ is a constant. From Eq. (10) we see no intermodulation terms

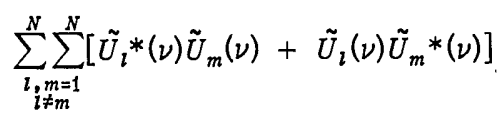

present as would be the case in coherent averaging of the filters.

Although the diffraction efficiency of coherent averaging (single exposure) is higher, as pointed out by LaMacchia, ${ }^{14}$ the simplicity of filter construction by incoherent averaging by far more than compensated the slightly lowered diffraction efficiency. The experimental results show good correlation peaks (see Fig. 5). 


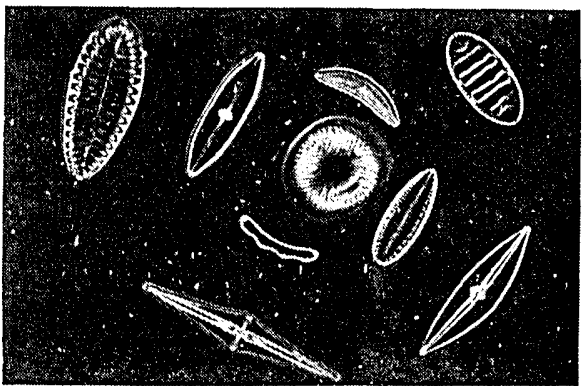

(a)

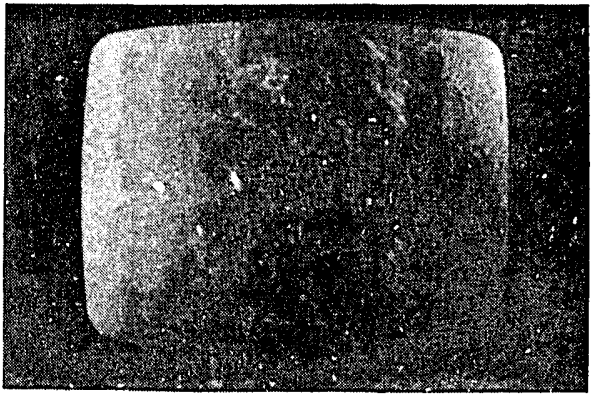

(b)

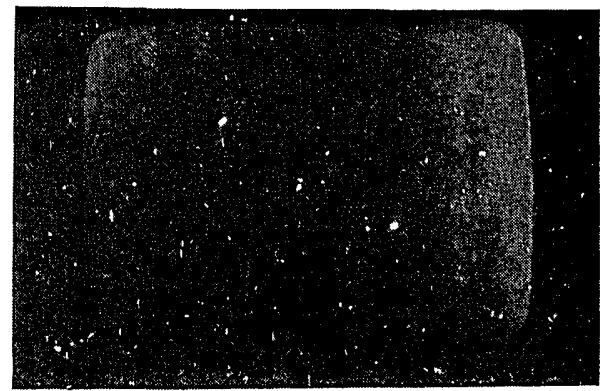

(c)

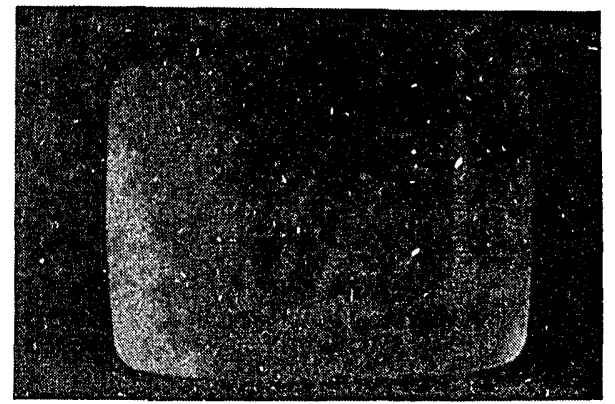

(d)

Fig. 4. (a) Diatoms used as input; (b), (c), (d), output correlation peaks for three pairs of different diatoms.

Averaged filters for size variations, for different depths of focus, or for both size variations and depths of focus can be realized by this method. Too many superpositions of filters, however, will cause a decrease in diffraction efficiency. One alternative to solving the depth of focus problem is to average the input patterns. (The detail will be given in another paper in the near future.)

The technique of averaged filtering can be carried further to the extent that one single averaged filter can be used to detect the presence of several different species of diatoms. In this case, we use incoherent averaging to record multiple filters for different species of diatoms. We have obtained good results for a multiple filter containing up to four individual filters. This method differs from the Vander Lugt multiple filter ${ }^{6,15}$ in that only a single detector is needed instead of a multiple detecting system. There will be some uncertainty, for example, if a multiple filter contains say two different component filters $\tilde{U}_{s 1}^{*}$, and $\tilde{U}_{s 2}^{*}$ is used to match the unknown input, and we detect two correlation peaks at the output plane. We only know there are three possibilities, but we are not sure which is the exact situation:

(1) two diatoms of $U_{s 1}$ presented in the unknown input;

(2) two diatoms of $U_{s 2}$ presented in the unknown input;

(3) one diatom $U_{s 1}$ and one diatom $U_{s 2}$ presented in the unknown input.

This seems to be confusing when dealing with character recognition or fingerprint recognition.

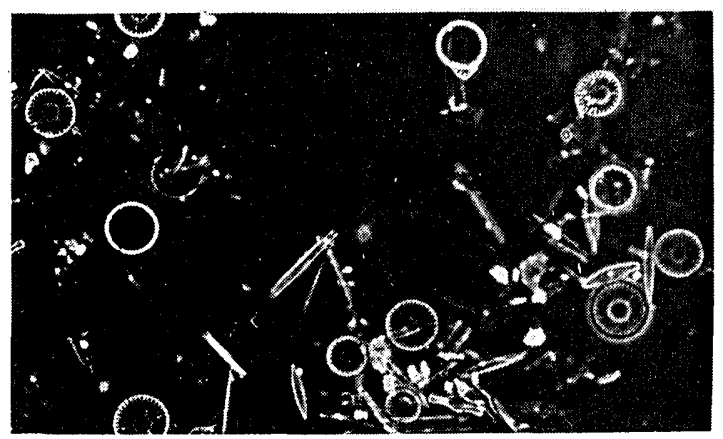

(a)

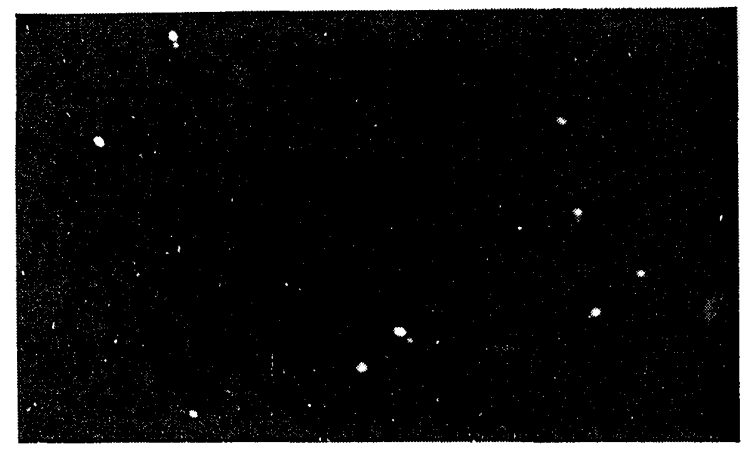

(b)

Fig. 5. (a) Poor quality input of diatom samples; (b) output correlation peaks indicating the presence of nine similar diatoms. 
However, in diatom recognition for water pollution monitoring, it is quite possible that the method is valuable, because it is the distribution of the diatoms that is important.

\section{Results and Conclusions}

Figure 3 shows a sample input (a) and three single signals detected (b), (c), (d). Shown in Fig. 4 is a photograph of a sample input (a) together with three pairs (b), (c), (d), of autocorrelation signals. Each pair of signals was obtained from a single filter that had two different diatoms incoherently (see Sec. IV) averaged. Another rather poor quality input sample is shown in Fig. 5(a) and consists of mostly the same type of diatoms, only with some size and depth of focus variation. Figure 5(b) shows nine autocorrelation signals indicating the success that the averaged filter (one filter with two diatoms incoherently averaged) had in identifying the nine similar diatoms. These results are, thus far, representative of the identification of diatoms via matched spatial filtering.

We are continuing to develop matched spatial filters for new diatoms as they are prepared from water samples. Once a large enough sample set of diatoms is studied with the optical processor, one can then statistically analyze the level of confidence for identification of diatoms via matched spatial filtering. Further studies on the orientation and size variation of diatoms are presently being conducted. In addition, a search is in progress to find an improved means of handling the input (i.e., the use of real-time read, write, and erasable capabilities).
The authors thank John Cairns, Jr., K. L. Dickson, and J. P. Slocomb for the biological input to this research. In particular we thank A. W. Lohmann and T. C. Strand for their valuable comments.

This research was supported in part by the National Science Foundation/RANN Division, U.S. Army Medical Research and Development Command, and the Environmental Protection Agency.

\section{References}

1. J. Cairns, Jr., K. L. Dickson, S. P. Almeida, et al., Arch. Mikrobiol. 83, 141 (1972).

2. J. Cairns, Jr., K. L. Dickson, S. P. Almeida, and J. K. T. Eu, in Trace Subs. in Environmental Health-8, D. Hemphill, Ed. (University of Missouri, Columbia, 1974), p. 223.

3. R. Patrick, M. H. Hohn, and J. H. Wallace, Proc. Nat. Acad. Sci. USA No. 259, 12 (1954).

4. R. Patrick, in Bio. Meth. for Assessment of Water Qual., ASTM STP 528, J. Cairns, Jr., and K. L. Dickson, Eds. (ASTM, Philadelphia, 1973), p. 76.

5. R. L. Kaesler and J. Cairns, Jr., Am. Midl. Nat. 88, No. 1, 56 (1972).

6. A. Vander Lugt et al., Optical and Electro-Optical Information Processing (MIT Press, Cambridge, 1965), pp. 125-141; A. Vander Lugt, IEEE Inf. Theory IT-10, 139 (1964).

7. J. E. Rau, J. Opt. Soc. Am. 56, 1490 (1966).

8. C. S. Weaver et al., Appl. Opt. 5, 1248 (1966); 9, 1672 (1970).

9. J. Armitage and A. Lohmann, J. Opt. Soc. Am. 54, 1404 (1964); Appl. Opt. 4, 461 (1965); A. Lohmann et al., Appl. Opt. 7, 651 (1968).

10. S. Iwasa and J. Feinleib, Opt. Eng. 13, No. 3, 235 (1974).

11. S. G. Lipson and P. Nisenson, Appl. Opt. 13, 2052 (1974).

12. A. Vander Lugt, Appl. Opt. 6, 1221 (1967).

13. J. C. Viénot et al., Appl. Opt. 12, 950 (1973).

14. J. T. LaMacchia et al., Appl. Opt. 7, 1857 (1968).

15. A. Vander Lugt, Appl. Opt. 5, 1760 (1966).

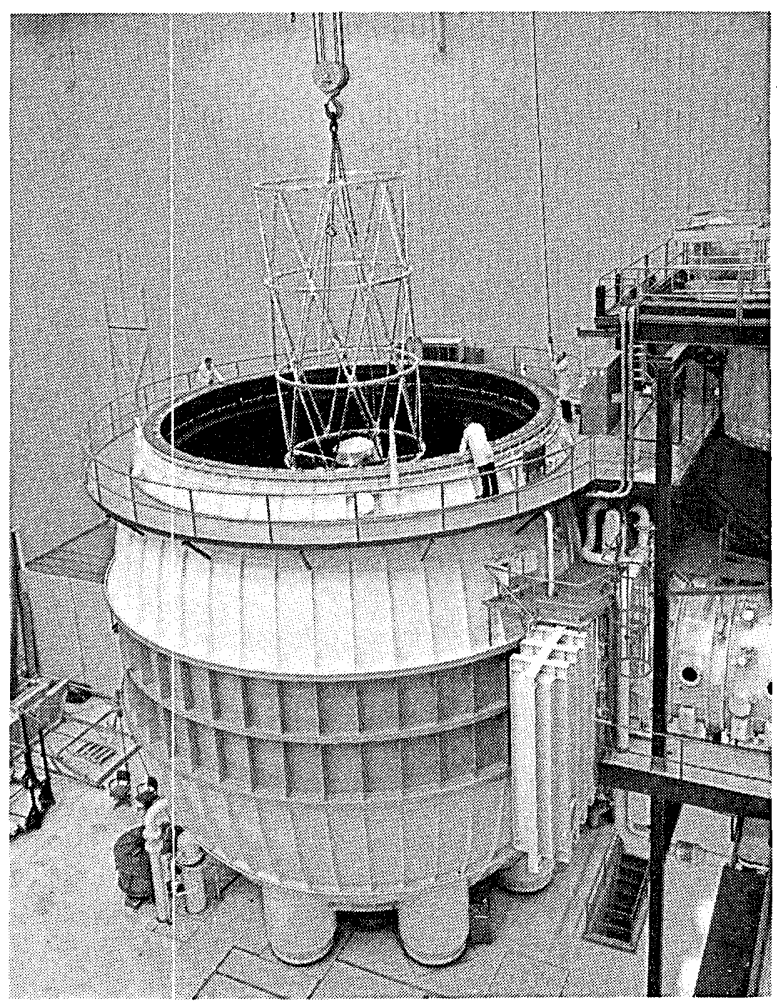

Boeing Aerospace Company engineers and technicians guide the graphite/epoxy metering truss for NASA's 2.4-m space telescope from the firm's large space vacuum chamber at the Boeing Space Center near Seattle, Washington. The truss, a prototype of the structure to hold the space telescope's mirrors, proved it could withstand the harsh environment of space without thermal distortions. 